\title{
SOME INEQUALITIES FOR SECTOR MATRICES
}

\author{
MinghuA LiN
}

Abstract. Two new inequalities are proved for sector matrices. The first one complements a recent result in [Oper. Matrices, 8 (2014) 1143-1148]; the second one is an analogue of the AMGM inequality, where the geometric mean for two sector matrices was introduced in [Linear Multilinear Algebra 63 (2015) 296-301]. As an application of the second inequality, we present similar inequalities for singular values or norms.

Mathematics subject classification (2010): 15A45, 15A42, 47A30.

Keywords and phrases: Inequality, singular value, unitarily invariant norm, geometric mean.

\section{REFERENCES}

[1] R. Bhatia, Positive Definite Matrices, Princeton University Press, Princeton, 2007.

[2] R. Bhatia, Matrix Analysis, GTM 169, Springer-Verlag, New York, 1997.

[3] S. DRURY, Principal powers of matrices with positive definite real part, Linear Multilinear Algebra 63 (2015) 296-301.

[4] S. DRURY, M. LIN, Singular value inequalities for matrices with numerical ranges in a sector, Oper. Matrices, 8 (2014) 1143-1148.

[5] C.-K. LI, N. SzE, Determinantal and eigenvalue inequalities for matrices with numerical ranges in a sector, J. Math. Anal. Appl. 410 (2014) 487-491.

[6] M. Lin, Extension of a result of Haynsworth and Hartfiel, Arch. Math. 104 (2015) 93-100.

[7] X. ZHAN, Inequalities involving Hadamard products and unitarily invariant norms, Adv. in Math. (China) 27 (1998) 416-422.

[8] F. ZHANG, A matrix decomposition and its applications, Linear Multilinear Algebra 63 (2015) 20332042.

[9] P. ZHANG, A further extension of Rotfel'd theorem, Linear Multilinear Algebra, 63 (2015), 2511-2517. 